\title{
Systematic computational identification of prognostic cytogenetic markers in neuroblastoma
}

\author{
Chao Qin ${ }^{1,2}$, Xiaoyan He ${ }^{3}$, Yanding Zhao ${ }^{4}$, Chun-Yip Tong ${ }^{2}$, Kenneth Y. Zhu ${ }^{5}$, Yongqi Sun ${ }^{*}$ and Chao Cheng ${ }^{2^{*}}$
}

\begin{abstract}
Background: Neuroblastoma (NB) is the most common extracranial solid tumor found in children. The frequent gain/loss of many chromosome bands in tumor cells and absence of mutations found at diagnosis suggests that NB is a copy number-driven cancer. Despite the previous work, a systematic analysis that investigates the relationship between such frequent gain/loss of chromosome bands and patient prognosis has yet to be implemented.
\end{abstract}

Methods: First, we analyzed two NB CNV datasets to select chromosomal bands with a high frequency of gain or loss. Second, we applied a computational approach to infer sample-specific CNVs for each chromosomal band selected in step 1 based on gene expression data. Third, we applied univariate Cox proportional hazards models to examine the association between the resulting inferred copy number values (iCNVs) and patient survival. Finally, we applied multivariate Cox proportional hazards models to select chromosomal bands that remained significantly associated with prognosis after adjusting for critical clinical variables, including age, stage, gender, and MYCN amplification status.

Results: Here, we used a computational method to infer the copy number variations (CNVs) of sample-specific chromosome bands from NB patient gene expression profiles. The resulting inferred CNVs (iCNVs) were highly correlated with the experimentally determined CNVs, demonstrating CNVs can be accurately inferred from gene expression profiles. Using this iCNV metric, we identified 58 frequent gain/loss chromosome bands that were significantly associated with patient survival. Furthermore, we found that 7 chromosome bands were still significantly associated with patient survival even when clinical factors, such as MYCN status, were considered. Particularly, we found that the chromosome band chr11p14 has high potential as a novel candidate cytogenetic biomarker for clinical use.

Conclusion: Our analysis resulted in a comprehensive list of prognostic chromosome bands supported by strong statistical evidence. In particular, the chr11p14 gain event provided additional prognostic value in addition to wellestablished clinical factors, including MYCN status, and thereby represents a novel candidate cytogenetic biomarker with high clinical potential. Additionally, this computational framework could be readily extended to other cancer types, such as leukemia.

Keywords: Cytogenetic marker, Neuroblastoma, Chr11p14, Chr11q23, Prognosis

\footnotetext{
* Correspondence: yqsun@bjtu.edu.cn; chao.cheng@bcm.edu

'Beijing Key Lab of Traffic Data Analysis and Mining, School of Computer

and Information Technology, Beijing Jiaotong University, No.3

Shangyuancun, Beijing 100044, Haidian District, China

2Department of Medicine, Baylor College of Medicine, BCM451, Suite 100D

Houston, TX 77030, USA

Full list of author information is available at the end of the article
}

(c) The Author(s). 2019 Open Access This article is distributed under the terms of the Creative Commons Attribution 4.0 International License (http://creativecommons.org/licenses/by/4.0/), which permits unrestricted use, distribution, and reproduction in any medium, provided you give appropriate credit to the original author(s) and the source, provide a link to the Creative Commons license, and indicate if changes were made. The Creative Commons Public Domain Dedication waiver (http://creativecommons.org/publicdomain/zero/1.0/) applies to the data made available in this article, unless otherwise stated. 


\section{Background}

Neuroblastoma (NB) is the most common extracranial solid tumor, usually occurring in early childhood, and it is also the third-most common cancer in babies after leukemia and brain cancer [1]. It is derived from primitive cells of the sympathetic nervous system and usually arises in the abdomen or chest [2]. Approximately 2550 cases occur per million individuals [3], and $90 \%$ of cases arise in children less than 5 years old [4].

To date, two widely used stage classification systems for NB patients have been developed to facilitate clinical research and improve the outcomes of children with NB. The International Neuroblastoma Staging System (INSS), developed in 1988, allows the classification of NB patients into Stage 1, 2A, 2B, 3, 4, and 4 S before surgical resection of the tumor [5]. The International Neuroblastoma Risk Group Staging System (INRGSS), proposed in 2009, allows the classification of NB into Stage L1, L2, M and MS based on the results of imaging tests (CT or MRI and MIBG scans) before surgery [6]. To help doctors select the optimal treatment [6], International Neuroblastoma Risk Group (INRG) combines INRGSS information, histologic category, MYCN status, and other factors to classify patients into low-, intermediate- and high-risk groups.

Prognostic markers are another important feature used to help predict the patient's clinical outlook [7-9]. For NB patients, many classic prognostic markers, such as age, tumor histology [10], DNA ploidy [11], transcription instability [12], and MYCN amplification [13-15] have been used to predict the prognostic outcome of patients. Among them, $M Y C N$ is the most critical prognostic marker in NB patients. $M Y C N$ is a master transcription factor that activates growth-sustaining genes and represses genes that drive differentiation [14]. MYCN amplification is found in approximately $25 \%$ of all tumors, and most malignant NB patients exhibit $M Y C N$ amplification [16].

Many studies report the gain of $1 \mathrm{q}, 2 \mathrm{p}$, and $17 \mathrm{q}$ along with a loss of $1 \mathrm{p}, 3 \mathrm{p}, 4 \mathrm{p}, 14 \mathrm{q}, 11 \mathrm{q}, 17 \mathrm{q}$, and $19 \mathrm{q}$ in the genomes of NB patients (reviewed in [17, 18]). Deletion of chr1p36 occurs in $23-35 \%$ of patients [19-21], and deletion of 11q23 occurs in $26-44 \%$ of patients [21-23]; each is associated with poor prognosis. The frequent chromosome segment gains and losses [24, 25] but few mutations have been found in NB tumor samples, suggesting that NB is a copy number-driven cancer $[17,26]$. Nevertheless, a systematic analysis for the identification of prognostic-associated chromosome bands with frequent gain/loss events for NB patients is still lacking and will be helpful for clinicians in treatment selection.

In this study, we took advantage of the abundant NB genomic data (gene expression data, copy number variation (CNV) data, and clinical information) to systematically identify chromosomal aberration events (gains or losses) that were associated with the clinical outcomes of
NB patients. Our analyses revealed a number of chromosomal bands that were frequently amplified or deleted in NB samples with significant associations at the prognostic level. Particularly, some bands (chr11q23, chr11p14) were still predictive of patient survival after adjusting for well-established clinical variables, including $M Y C N$ amplification status, an extremely widely used prognostic biomarker. These chromosomal aberrations have the potential to be developed into effective cytogenetic markers, as they are visible by microscopic examination. Such a marker can further improve prognostic prediction and patient stratification in NB. Moreover, the computational framework introduced in this article can be readily applied to the identification of cytogenetic markers in other cancer types.

\section{Methods}

Dataset and data processing

NB gene expression datasets and related clinical data with sufficient overall outcome information were downloaded from the Gene Expression Omnibus (GEO) under accession number GSE62564 ( $\mathrm{Su}$ et al., $n=498$ ). The International Cancer Genome Consortium (ICGC) data portal was accessed under the code NBL-US (Pugh et al., $n=$ 249), which contained the segmental chromosome CNV data. The European Bioinformatics Institute was accessed under ID: E-MTAB-179 (Oberthuer et al., $n=478$ ). The other dataset used in this study that did not contain survival information was downloaded from the GEO under accession number GSE45478 (Kocak et al., $n=123$ ) with the NB segmental CNV information and gene expression profiles. Among them, GSE62564 was generated using the RNA-seq platform, NBL-US and E-MTAB-179 using a one-channel microarray platform, and GSE45478 using a two-channel array platform. A total of 1347 samples were included in these datasets. A summary of these four datasets is provided in Additional file 8: Table S8. The gene expression values obtained from the RNA-seq platform and the one-channel microarray platform were log transformed, and gene-wise mean normalization was performed to obtain the relative expression values for these datasets. The genes associated with positional gene set data were downloaded from the $\mathrm{C} 1$ collection of MSigDB (http://software.broadinstitute.org/gsea/msigdb/index.jsp) [27], all bands from the $X$ and $Y$ chromosomes were excluded.

\section{Mapping the segmental chromosome's CNV to the chromosome band's CNV}

For each sample, the segmental chromosome's CNV was mapped to the chromosome band's CNV. The CNV of chromosome band $i$ is defined as follows:

$$
C N V(i)=\sum_{j=1}^{n} \frac{l_{i j}}{l_{i}} * S C N V(j),
$$


where $S C N V(j)$ is the CNV value of the $j$ th segment chromosome, $l_{i}$ is the length of chromosome band $i, l_{i j}$ is the length of the overlap between the chromosome band $i$ and the segmental chromosome $j$, and $n$ is the number of the segmental chromosomes in a given sample.

\section{Calculation of chromosome band CNV (iCNV) based on the gene expression data}

For a given gene expression dataset, all the genes located on a given chromosome band were grouped into a set designated ' $\mathrm{B}$ ', and the rest of the genes located on any other chromosome bands were grouped into a set designated 'A'. The inferred CNV for a given chromosome band is the value of Student's $t$ statistic comparing the gene sets $\mathrm{B}$ and $\mathrm{A}$ :

$$
\mathrm{t}=\frac{\overline{x_{B}}-\overline{x_{A}}}{\sqrt{s_{B}^{2} / n_{B}+s_{A}^{2} / n_{A}}}
$$

where $\bar{x}$ is the mean, $s^{2}$ is the variance, and $n$ is the number of genes located in a gene set [28]. For each sample in the dataset, this process was iterated for each chromosome band such that we obtained a matrix of iCNVs for each chromosome band of each sample. If the number of genes located on a chromosome band was less than 10 , we considered the Student's $t$ statistic measurement unreliable, and that chromosome band was eliminated from further analysis.

\section{Survival analysis}

A univariate Cox proportional hazards model was fitted to the iCNV for each chromosome band across all samples in a dataset to evaluate the relationship between $\mathrm{iCNV}$ and sample survival time. For survival-associated iCNVs of chromosome bands, multivariate Cox proportional hazards models were used to examine prognostic abilities, while potential confounding factors, including $M Y C N$ status, age, gender, and stage were considered. Kaplan-Meier curves were used to visualize the results from the Cox proportional hazards model. Specifically, the iCNVs were stratified into two groups by the median value for generating the Kaplan-Meier curves.

All survival analyses were conducted in $\mathrm{R}$ using the "survival" package. Specifically, "coxph", "survfit", and "surdiff" were called to create the Cox proportional hazards model, plot Kaplan-Meier curves, and compare the two survival curves.

\section{Results}

\section{Overview of this study}

To systematically identify novel candidate cytogenetic biomarkers, we performed an integrative analysis via the following main steps (Fig. 1). First, we analyzed two NB
$\mathrm{CNV}$ datasets to select chromosomal bands with a high frequency of gain or loss. One of the datasets included samples from all stages [29], while the other included only high-risk NB samples (mostly Stage IV) [30]. By combining results from both datasets, we selected 223 chromosomal bands with $>15 \%$ gain/loss frequency in at least one of the datasets. Second, we applied a computational approach to infer sample-specific CNVs for each chromosomal band selected in step 1 based on gene expression data. This approach was applied to $\mathrm{Su}$ [31] and Oberthuer [32] NB datasets, which contained both gene expression profiles and clinical information, particularly the survival times of patients. Third, we applied univariate Cox proportional hazards models to examine the association between the resulting inferred copy number values (iCNVs) and patient survival. Finally, we applied multivariate Cox proportional hazards models to select chromosomal bands that remained significantly associated with prognosis after adjusting for critical clinical variables, including age, stage, gender, and $M Y C N$ amplification status. Of note, we used gene expression data rather than CNV data to determine the association between chromosomal bands and patient survival. We selected gene expression data because more gene expression data are available with a significantly larger sample size and higher quality of survival information than CNV data, thus ensuring sufficient statistical power and rigorous results of our analysis.

\section{CNV data analysis identified chromosome bands with a high frequency of gain or loss}

To identify chromosome bands that were frequently amplified or deleted in NB patients, we analyzed two CNV datasets. Each dataset identified chromosomal segments with abnormal copy numbers determined using array-based comparative genomic hybridization and genome-wide human single nucleotide polymorphism (SNP) arrays 6.0, respectively. One dataset contained 122 samples from different stages (the Kocak dataset) [29], and the other dataset focused on high-risk NB samples, including 149 out of 150 from Stage IV tumors (the Pugh dataset) [30].

By combining the two datasets, our analysis included chromosome bands with high gain/loss frequency generally in all samples and in high-risk samples. Specifically, for each sample, we mapped the aberrant segments to chromosome bands and obtained copy number values of each band (see Additional file 1: Table S1). In Fig. 2a and $b$, we summarized the frequency of gain/loss for each chromosome band (272 in total excluding the $\mathrm{X}$ and $\mathrm{Y}$ chromosomes) in the two datasets. As expected, there was a negative correlation between amplification and deletion frequency. Namely, a set of chromosome bands was frequently amplified in NB with high frequency, while a different set of bands was frequently 
A

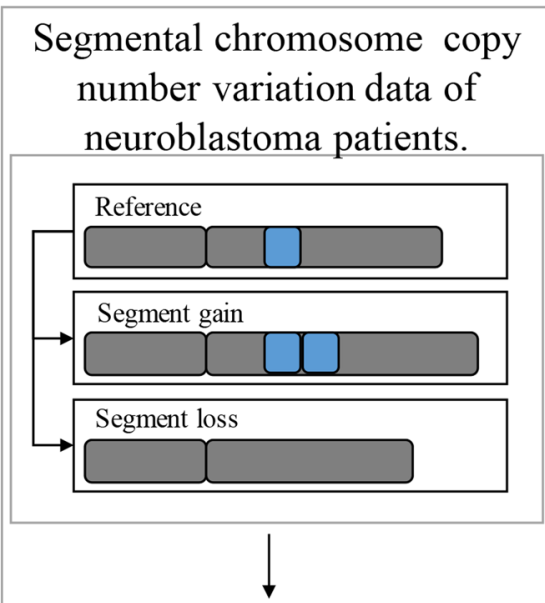

Chromosome bands copy number variation profile



High frequent gain/loss chromosome bands

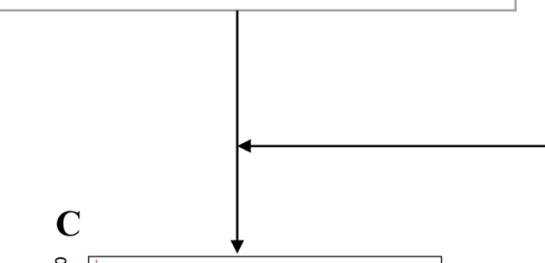

B

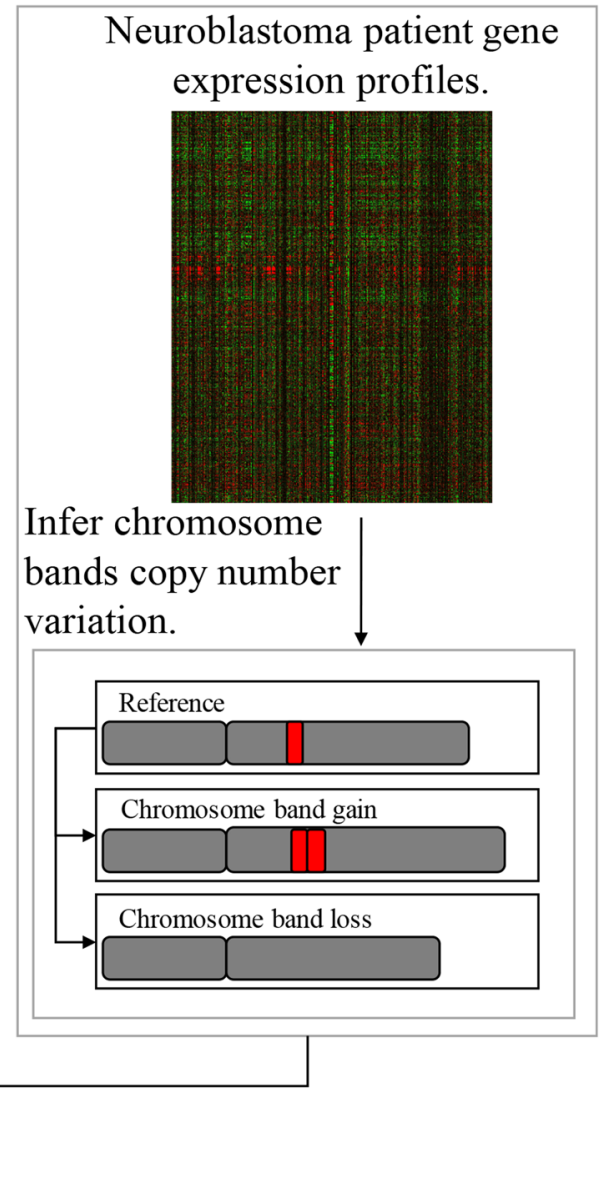

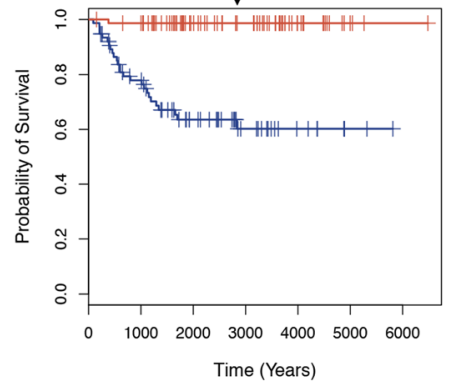

Frequent gain/loss

chromosome bands associated with survival.

Fig. 1 Schematic diagram of our analysis. a The segmental chromosome copy number variation data of neuroblastoma patients was used to map to the chromosome band copy number variation, and the frequency of chromosome band gain/loss was obtained. We selected chromosome bands with a gain/loss frequency $>15 \%$ as the frequent gain/loss chromosome bands. b The neuroblastoma patient gene expression profiles were used to calculate the inferred copy number variation (iCNV) of each chromosome band. c A Cox proportional hazard model was used to measure the correlation between frequent gain/loss chromosome band iCNVs and patient survival time

deleted. This enabled us to define frequently amplified and frequently deleted chromosome band sets. By setting the cut-off values to $15 \%$, we identified 118 frequently amplified bands and 96 frequently deleted bands in the Kocak dataset and 70 frequently amplified bands and 61 frequently deleted bands in the Pugh dataset (Fig. 2c).

Although the observed gain/loss frequency was highly consistent, some chromosome bands had notable differences between the two datasets, indicating variations in chromosome aberration events among tumor stages. For example, chr1p36 was lost in $16 \%$ of samples in the Kocak dataset but was lost in as high as $36 \%$ of Stage IV samples (the Pugh dataset). To obtain a comprehensive list of chromosome bands with high frequency gain/loss events, we took the union of the bands identified from the two datasets, yielding 125 and 105 frequently amplified and frequently deleted chromosome band sets, respectively (Fig. 2c) (Additional file 2: Table S2). Most 


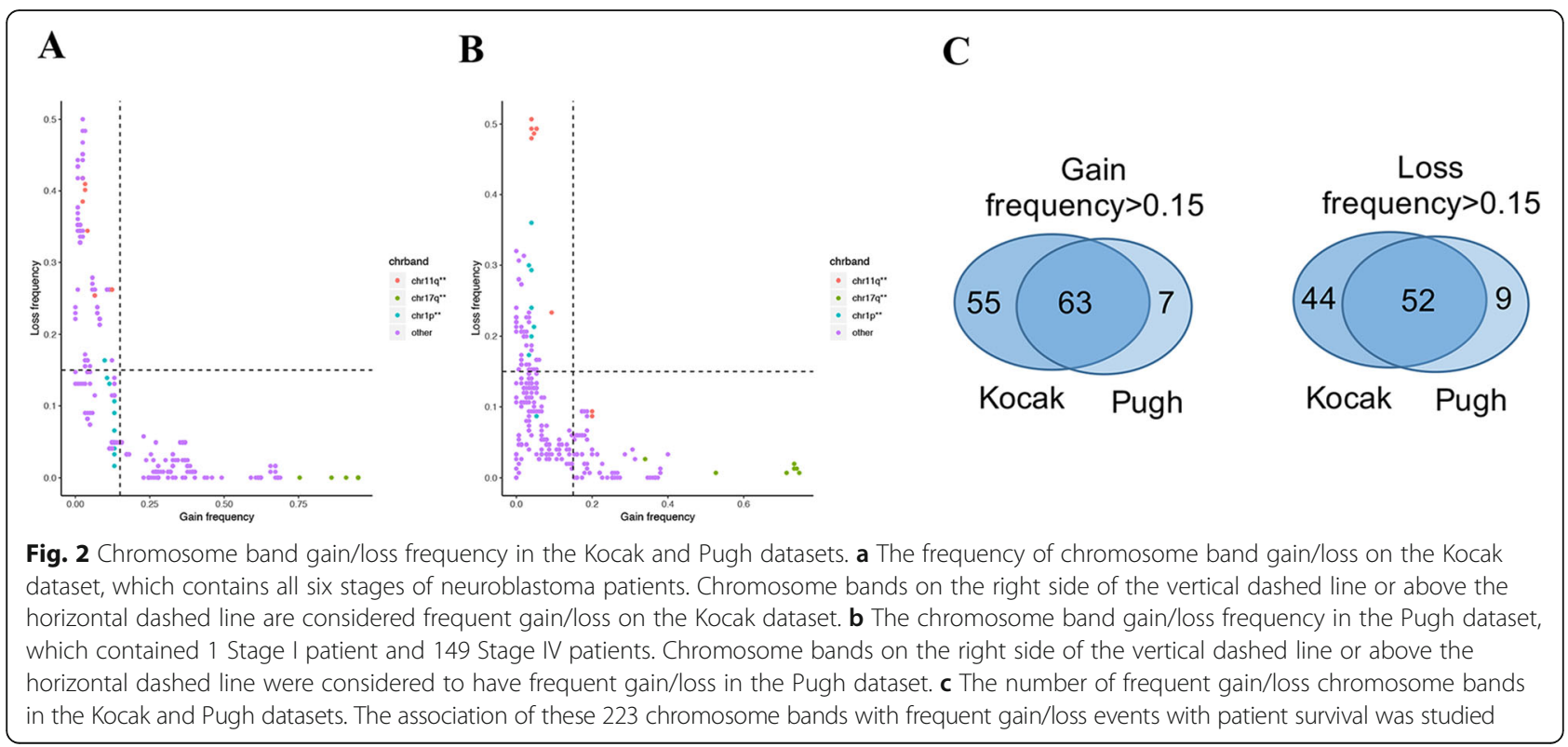

of the previously established cytogenetic markers are included in our band sets. For example, the well-known chr17q21 [33, 34] was amplified in 95\% (the Kocak dataset) and $74 \%$ (the Pugh dataset) of samples, while chr11q23 [35, 36] was deleted in 40\% (the Kocak dataset) and $49 \%$ (the Pugh dataset) of samples.

Interestingly, between the frequently amplified and frequently deleted chromosome band sets, there were 7 overlapping chromosome bands. These 7 chromosome bands were chr11p15, chr11p14, chr11p13, chr11p12, chr11p11, chr11q11, and chr11q12. In the Kocak dataset, they were more likely to be deleted. In the Pugh dataset, they were more likely to be amplified. It is notable that all these chromosome bands are from chromosome 11 . Thus, we examined the co-occurrences of the grain/loss events of these bands in samples from the Kocak and Pugh datasets (Additional file 9: Figure S1 and S2). Out of the 60 samples in the Kocak dataset, 34 are associated with whole-chromosome gain $(n=3)$ or loss $(n=31)$ of chromosome 11,45 with whole-arm gain $(n=13)$ or loss $(n=32)$ of chromosome $11 \mathrm{p}$, and 34 with whole-arm gain $(n=3)$ or loss $(n=31)$ of chromosome 11q. Out of the 167 samples in the Pugh dataset, 43 are associated with whole-chromosome gain $(n=7)$ or loss $(n=36)$ of chromosome 11, 90 with whole-arm gain $(n=48)$ or loss $(n=42)$ of chromosome $11 \mathrm{p}$, and 45 with whole-arm gain $(n=7)$ or loss $(n=38)$ of chromosome 11q.

We noted that many of these chromosome bands were consecutive in the genome, e.g., chr1p36, chr1p35, chr1p34, and chr1p33. The chromosome bands from the same clusters were likely associated with the same chromosome gain/loss hot spot. Thus, we produced a list of nonredundant chromosome bands by selecting the most frequently gained or lost bands from each cluster, resulting in a total of 29 frequently amplified and 29 frequently deleted nonredundant bands (Additional file 3: Table S3).

\section{Copy numbers of chromosome bands can be accurately inferred from gene expression data}

To identify cytogenetic events of potential prognostic value, we aimed to examine the association between chromosome band gain/loss events and patient survival in a systematic manner. The above-described Kocak CNV dataset did not provide patient survival information and therefore could not be used for this analysis. The Pugh $\mathrm{CNV}$ dataset comprised mostly high-risk patients. However, a few high-quality gene expression datasets from NB patients were generated, which provided expression profiles for a large number of tumor samples and carefully prepared survival information (Additional file 4: Table S4). Therefore, we adapted a previously proposed method [28] to infer the copy number of chromosome bands based on these high-quality gene expression datasets. This method compared the expression of genes located in a chromosome band against other genes and used the Student's $t$ statistic to infer the chromosome band status. This analysis resulted in an inferred copy number value (iCNV) for each chromosome band in each tumor sample.

First, we assessed the performance of this method by comparing the iCNVs to experimentally measured copy number scores using the Kocak dataset. As shown in Fig. 3a, we selected three samples with amplified, normal and deleted chr1p36. The expression of genes in this chromosome band reflected the band status with high fidelity. The iCNVs for this band were also highly correlated $(R=0.97)$ with the experimentally determined copy 


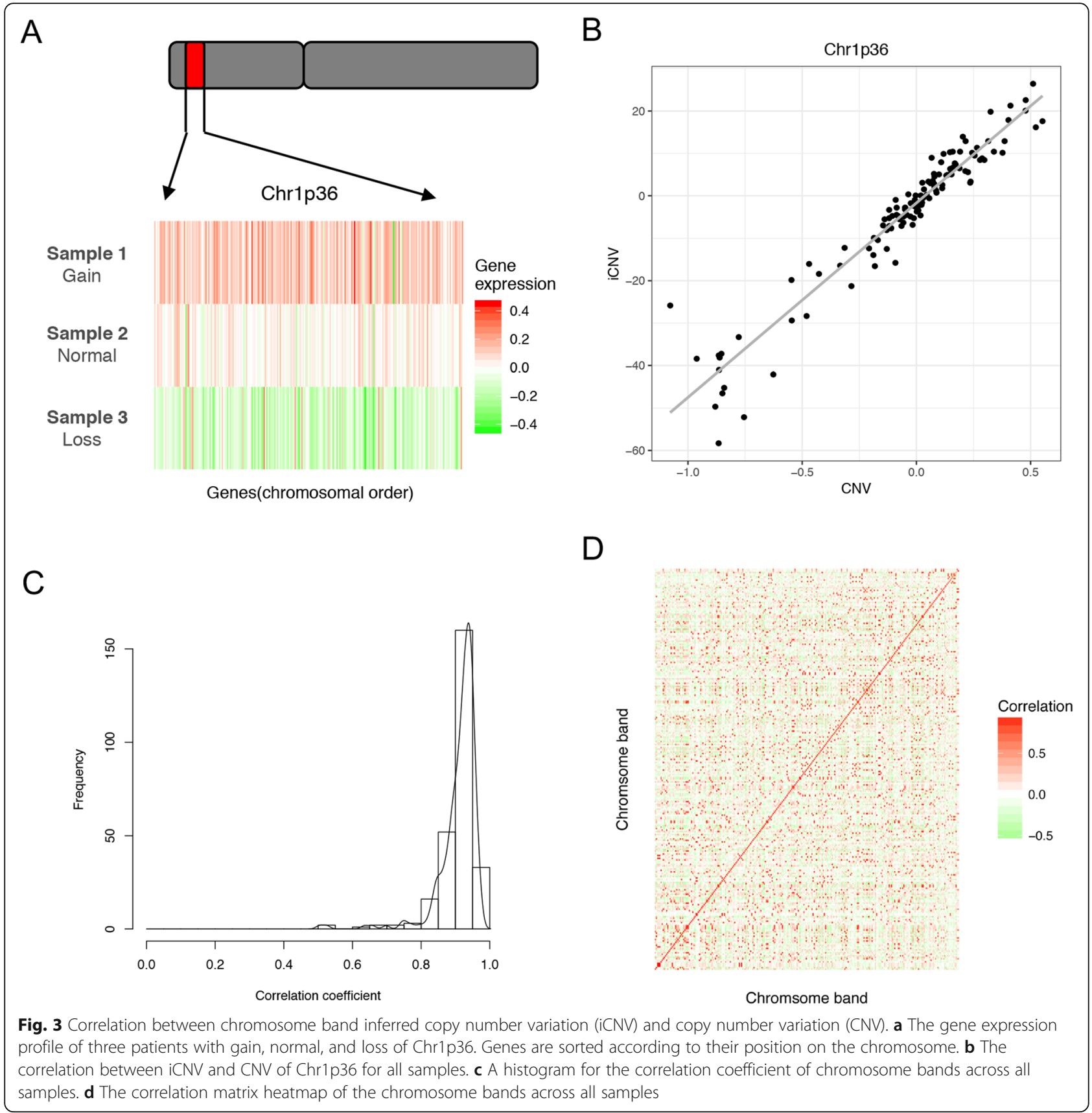

numbers (Fig. 3b). We then calculated the correlation coefficients for all of the 272 chromosome bands, and their distribution indicated a high accuracy of the copy number inference (Fig. 3c). More than $80 \%$ of chromosome bands had $\mathrm{R}>0.8$. Moreover, iCNVs for any particular band had the highest correlation with the measured copy number scores of the same band but had much lower correlations with other bands, indicating high specificity (Fig. 3d). Taken together, our results suggested that the iCNV inferred from gene expression data provided an accurate estimation of chromosome band copy numbers.
Identification of chromosome bands associated with patient prognosis

We then applied the chromosome band copy number inference method to two gene expression datasets, Su [31] and Oberthuer [32], to calculate sample-specific iCNVs. Subsequently, we examined the iCVNs for their association with prognosis. We focused our analysis on the 125 frequently amplified and 105 frequently deleted chromosome bands. We used univariate Cox proportional hazard models to identify chromosome bands that were significantly associated with patient survival. As 
shown in Fig. 4a, the gain/loss status of the 223 chromosome bands and the prognostic association with patient survival for the two datasets are briefly described. To obtain a list of prognostic chromosome bands of highest confidence, we combined the results from these two datasets and selected the bands that were significant in all datasets (adjusted $p<0.05$ ) (Fig. 4b). This combination of results yielded a total of 58 significant chromosome bands (Additional file 5: Table S5). Among the 27 frequently amplified bands, 24 were associated with good survival $(\mathrm{HR}<1)$, and three were associated with poor survival $(H R>1)$. Among the 31 frequently deleted bands, 22 were associated with good survival $(\mathrm{HR}<1)$, and 9 were associated with poor survival $(\mathrm{HR}>1)$ (Fig. 4c). Detailed information on the 58 prognostic-associated bands is shown in Fig. 4d. Most of the chromosomal bands with aberration events were clearly associated with good prognosis $(46 / 58)$. Furthermore, we found that most of these bands were either hot spots or near the hot spots, suggesting the importance of each hot spot area in terms of patient prognosis. We further selected four chromosome bands as examples, as shown in Fig. 5a, b. The loss of chr1p36 was associated with poor prognosis in both the $\mathrm{Su}$ and Oberthuer datasets, and this finding was consistent with previous reports $[37,38]$. We also found that the loss of chr14q22 was associated with good prognosis. The gains of chr12q22 and chr7q31 were associated with poor prognosis and good prognosis, respectively.

It is important to note that the status of $M Y C N$, the best-characterized genetic marker associated with poor prognosis in NB [14], may confound our results. To further examine whether the prognostic association of these

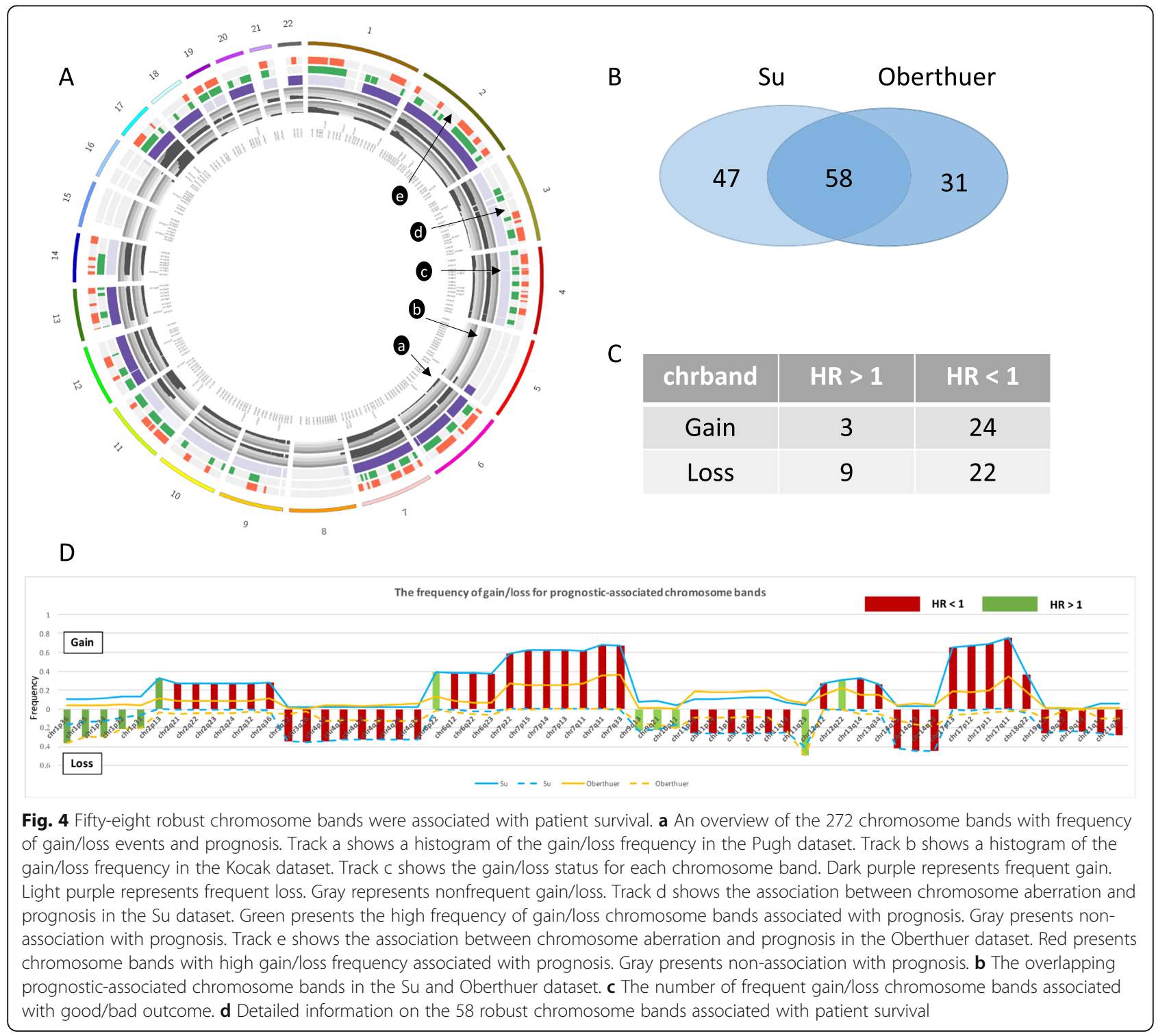




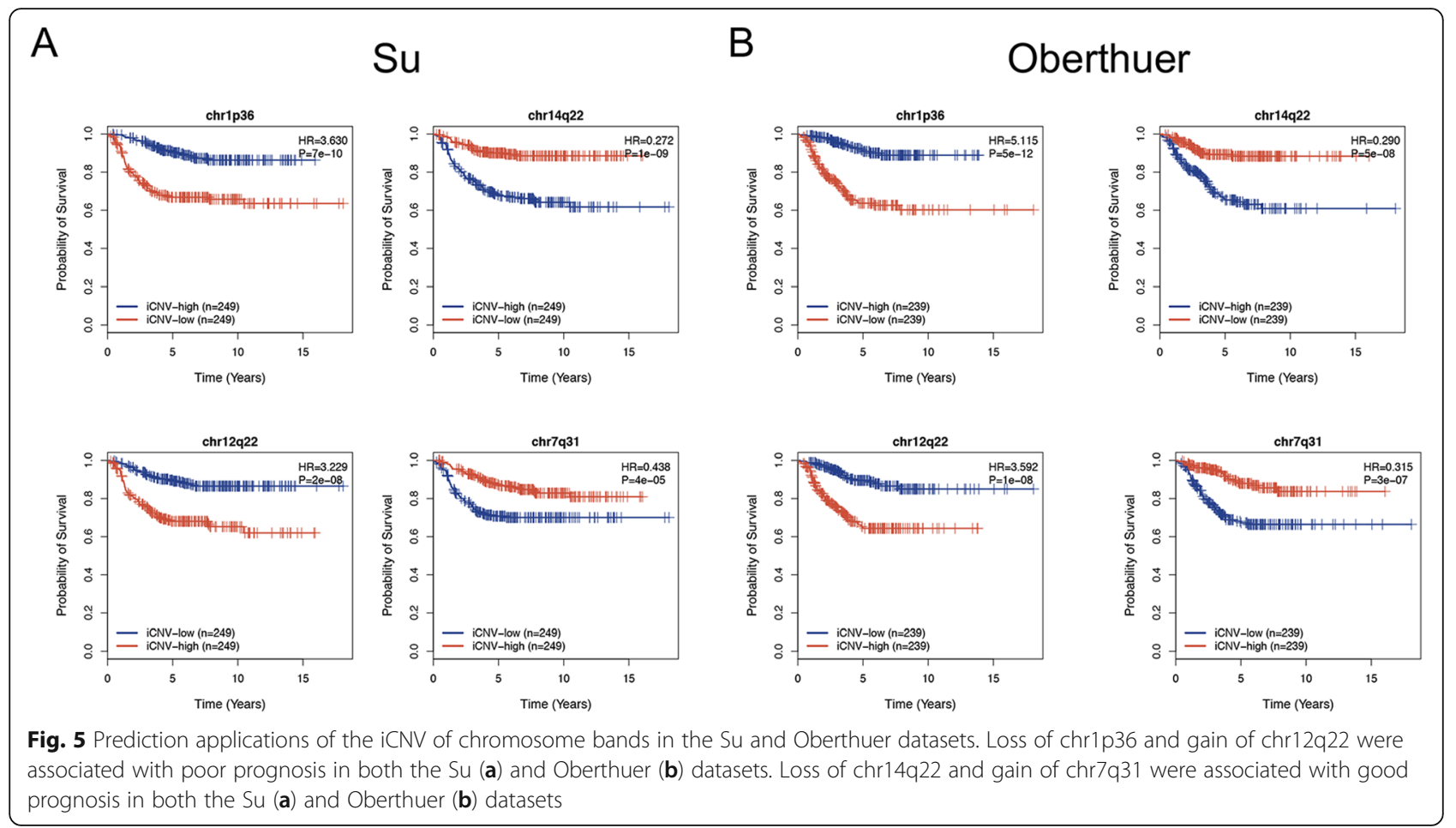

58 chromosome bands was independent of this clinical variable, we applied multivariate Cox proportional hazard models to the $\mathrm{Su}$ and Oberthuer datasets. The models included sample-specific iCNV for each band, stage, age, gender and status of $M Y C N$ status as covariates. We selected the chromosome bands with Cox proportional hazards $p$-value $<0.05$ in these two datasets which yielded 7 chromosome bands with iCNV associated with patient survival (Additional file 6: Table S6).

Taking the well-known cytogenetic marker chr11q23 as an example in the Su dataset, loss of chr11q23 was associated with poor prognosis, which was also consistent with a previous report [35] (Fig. 6a). After we considered potential confounding clinical variables including $M Y C N$ status, stages, sex, and age, using a multivariate Cox proportional hazard model, the iCNV for chr11q23 remained significant $(p=2 \mathrm{e}-04)$ with a hazard ratio of 1.13 (Fig. 6b). To further examine whether the iCNV for chr11q23 was still significant with patient prognosis in both the $M Y C N$ amplification patient cohort and the MYCN nonamplification patient cohort, we compared the survival curves of four groups stratified based on the level of iCNV and $M Y C N$ status (Fig. 6c). The iCNV for chr11q23 was still significantly associated with poor prognosis in the MYCN nonamplification patient cohort $(p=3 \mathrm{e}-06, \mathrm{HR}=4.49)$ while was less significant in the $M Y C N$ amplification patient group. We also performed these analyses on the Oberthuer dataset and obtained similar results (Fig. 6d, e, f).

In addition to chr11q23, among the seven chromosome bands, we found that chr11p14 was also a potential novel cytogenetic marker for measuring the progress of NB patients. Chr11p14 was an interesting chromosome band that exhibited a character different from most of the other chromosome bands. It exhibited not only loss events but also gain events. The frequency of gain events in the Pugh dataset was $18 \%$, but the frequency of loss events in the Kocak dataset was $26 \%$. As shown in Fig. $7 \mathrm{a}$, the gain events for chr11p14 were significantly associated with poor prognosis $(\mathrm{p}=3 \mathrm{e}-10, \mathrm{HR}=3.905)$ in the Su dataset. Furthermore, a significant result was obtained upon applying the multivariate Cox proportional hazard model, as described above (Fig. $7 \mathrm{~b}, \mathrm{p}=1 \mathrm{e}-04, \mathrm{HR}=$ 1.337). Additionally, among the $M Y C N$ nonamplification patients, the gain of chr11p14 was significantly associated with poor prognosis (Fig. $7 \mathrm{c}, \mathrm{p}=5 \mathrm{e}-06$, $H R=4.151$ ). To further validate our results, we performed the same analysis on the Oberthuer dataset and obtained similar results (Fig. $7 \mathrm{~d}$, e, f).

The Pugh dataset differs from the two datasets described above; most of the samples in this dataset are Stage IV (216 Stage IV, 1 Stage III and 30 Stage I). To further investigate the association between chromosome band gain/loss and high-risk status for patients, we applied a univariate Cox proportional hazard model and found 10 prognosis-associated chromosome bands (adjust $p<0.05$ ) (Additional file 7: Table S7). Seven of the 10 bands were also discovered in the other two datasets (Su and Oberthuer datasets). The other 3 chromosome bands were discovered in either the $\mathrm{Su}$ dataset or the Oberthuer dataset. 


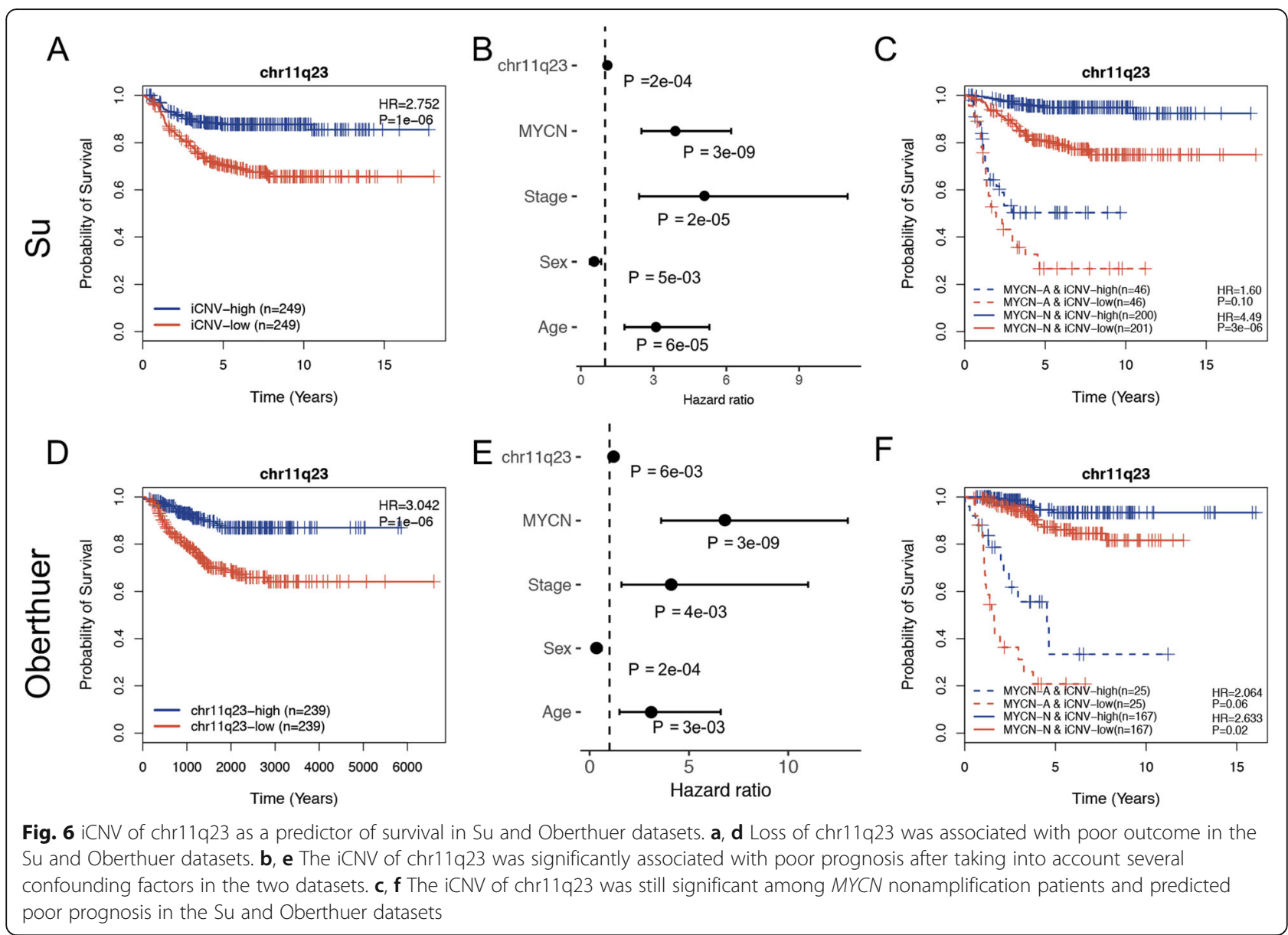

Taken together, our analysis resulted in a comprehensive list of prognostic-associated chromosome bands. In particular, the chr11p14 gain event provided additional prognostic value in addition to well-established clinical factors, including $M Y C N$ status, and thereby represents a novel candidate cytogenetic biomarker with high clinical potential.

\section{Discussion}

Chromosomal instability is a hallmark of human cancer and plays an important role during tumorigenesis and progression [39]. Frequent gain or loss of particular chromosome regions has been investigated in certain cancer types, such as leukemia and NB [40,41]. Some of these recurrent events have been developed into cytogenetic markers to define cancer subtype, predict prognosis, and select effective therapeutic interventions [42]. A list of chromosome aberration events in NB was previously compiled [17]. For example, deletion of chr1p36 [19] and chr11q23 [21] have been reported in 23-35\% and $26-44 \%$ NB samples, respectively. Both deletions were associated with poor prognosis. Conversely, whole gain of chr17q is associated with good prognosis [34].
Despite the important clinical implications and extensive reports in previous studies, the association of chromosome bands with prognosis has never been investigated in a systematic manner. In this study, we integrated different genomic data with clinical information to systematically identify novel candidate cytogenetic markers for improving NB prognosis.

We utilized two CNV datasets to identify chromosome bands with a high frequency of gain or loss events in NB. This could theoretically also be achieved by examining the $\mathrm{iCNV}$ inferred from gene expression data. The $\mathrm{iCNV}$ is an essential $t$ statistic that compares the relative expression levels of genes in a chromosome band with those not in that chromosome band. As such, each iCNV was associated with a $p$-value that could be estimated by referring to the $t$ distributions. Given an NB gene expression dataset, we might calculate samplespecific iCNVs along with corresponding $p$-values and then count the number of samples in which a band shows significantly higher (gain) or lower (loss) iCNV. However, the frequency of a gain/loss event can only be correctly calculated if the relative expression levels of genes were calculated by normalizing against their 


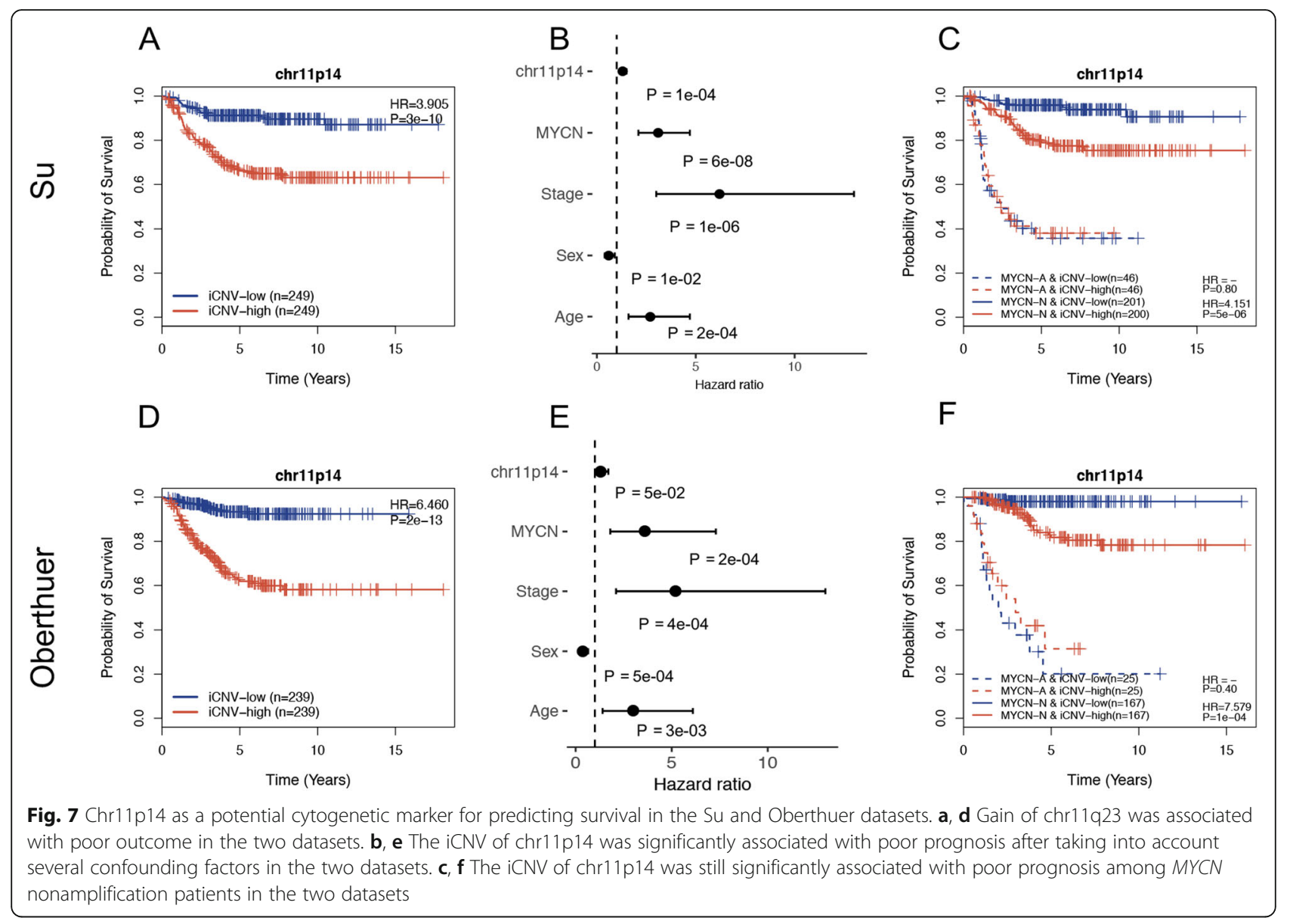

expression in normal tissue. Unfortunately, no normal control was available in the NB gene expression datasets. Therefore, in our analyses, we used the median expression of genes in all samples to convert absolute gene expression values to relative expression values, which has been proposed in [12]. For this reason, the iCNV calculated from these datasets indicated the copy numbers relative to the median reference rather than a normal control and did not correctly inform the gain/loss frequency of chromosome bands. On the other hand, the iCNV remained effective for examining the association with patient prognosis.

The CNV datasets were not used in our analysis to determine the association of chromosome band gain/loss events with prognosis because of their limitations. The Kocak dataset did not provide survival information, and the Pugh dataset contained only Stage IV NB samples (expect for 1 Stage III sample). In contrast, the gene expression datasets used in our analysis were originally produced for prognostic studies and had large sample sizes. For example, the $\mathrm{Su}$ dataset was generated by the SEQC Project, containing 498 carefully selected NB samples with detailed clinical information, including patient survival. In addition, the $\mathrm{Su}$ and Oberthuer datasets contained samples from all stages. Combined with the high risk-specific Pugh dataset, these gene expression datasets enabled us to systematically identify chromosome bands that were prognostic in all NB patients or found specifically in high-risk (most Stage IV) patients. Moreover, by combining results from multiple independent datasets, we expected to obtain a list of highly confident prognosticassociated chromosome bands for cytogenetic marker development.

$M Y C N$ amplification correlates with high-risk disease, has been found in $\sim 25 \%$ of NB patients, and is widely used as the most critical prognostic marker. For highrisk NB patients, the 5-year survival rate is approximately 40 to $50 \%$. After considering the $M Y C N$ status, we still found 7 chromosome bands significantly associated with patient survival. Chr11q23 has been well studied. However, gain of chr11p14 was significantly associated with poor prognosis, which has the potential to be a novel cytogenetic biomarker. In the high-risk group (Pugh dataset), we identified 10 chromosome bands associated with patient outcomes that were also found in the other two datasets. 


\section{Conclusions}

In conclusion, we performed a systematic analysis that integrated different genomic datasets with clinical information to identify chromosome band gain or loss events associated with NB patient prognosis. Our analysis resulted in a comprehensive list of prognostic chromosome bands supported by strong statistical evidence. In particular, the chr11p14 gain event provided additional prognostic value in addition to well-established clinical factors, including $M Y C N$ status, and thereby represents a novel candidate cytogenetic biomarker with high clinical potential. Additionally, the computational framework introduced in this article could be readily extended to other cancer types, such as leukemia.

\section{Supplementary information}

Supplementary information accompanies this paper at https://doi.org/10. 1186/s12920-019-0620-6

\section{Additional file 1: Table S1. The copy number values of each chromosomal band on the Kocak dataset and the Pugh dataset.}

Additional file 2: Table S2. A comprehensive list of chromosome bands with high frequency gain/loss events.

Additional file 3: Table S3. A list of nonredundant chromosome bands by selecting the most frequently gained or lost bands from each cluster.

Additional file 4: Table S4. The detailed information of 3 NB datasets. Additional file 5: Table S5. The combination of results yielded a total of 58 significant chromosome bands associated with patient prognosis.

Additional file 6: Table S6. Seven chromosome bands associated with patient survival after considering the confounding factors.

Additional file 7: Table S7. Ten prognosis-associated chromosome bands on the Pugh dataset.

Additional file 8: Table S8. Dataset summary.

Additional file 9: Figure S1. The landscape of chromosome bands gain/loss on Kocak dataset. Figure S2. The landscape of chromosome bands gain/loss on Pugh dataset.

\section{Abbreviations}

CNV: Copy number variation; GEO: Gene Expression Omnibus;

ICGC: International Cancer Genome Consortium; INRG: International Neuroblastoma Risk Group; INRGSS: International Neuroblastoma Risk Group Staging System; INSS: International Neuroblastoma Staging System; NB: Neuroblastoma; SNP: Single nucleotide polymorphism

\section{Acknowledgements}

Not applicable.

\section{Authors' contributions}

CC and YQS conceived the problem and managed the study. CQ and CC developed algorithms. CQ performed data analysis. CQ and CC wrote the manuscript. CC, XYH, YDZ, CYT, YQS and KYZ helped to analyze the data and edit the manuscript. All the authors read and approved the final manuscript.

\section{Funding}

This research was supported by the National Natural Science Foundation of China (NSFC 61572005, 61672086, 61702030, 61771058, 61272004), American Cancer Society (IRG-82-003-30), National Center for Advancing Translational Sciences of the National Institutes of Health (KL2TR001088), Fundamental Research Funds for the Central Universities (K18JB00060), and China Scholarship Council. The funding bodies played no role in the design of the study and collection, analysis, and interpretation of data and in writing the manuscript.

\section{Availability of data and materials}

The datasets analyzed during the current study are available from the Gene Expression Omnibus (GEO) under accession number GSE62564, GSE45478, from The International Cancer Genome Consortium (ICGC) data portal under the code NBL-US, and from The European Bioinformatics Institute under ID: E-MTAB-179. The genes associated with positional gene set data were downloaded from the C1 collection of MSigDB (http://software.broadinstitute.org/ gsea/msigdb/index.jsp), all bands from the $X$ and $Y$ chromosomes were excluded.

Ethics approval and consent to participate

Not applicable.

\section{Consent for publication}

Not applicable.

\section{Competing interests}

The authors declare that they have no competing interests.

\section{Author details}

${ }^{1}$ Beijing Key Lab of Traffic Data Analysis and Mining, School of Computer and Information Technology, Beijing Jiaotong University, No.3

Shangyuancun, Beijing 100044, Haidian District, China. ${ }^{2}$ Department of Medicine, Baylor College of Medicine, BCM451, Suite 100D, Houston, TX 77030, USA. ${ }^{3}$ Center for Clinical Molecular Medicine, Children's Hospital, Chongqing Medical University, Ministry of Education Key Laboratory of Child Development and Disorders, Key Laboratory of Pediatrics in Chongqing, Chongqing International Science and Technology Cooperation Center for Child Development and Disorders, Chongqing 400014, China. ${ }^{4}$ Department of Biomedical Data Science, Geisel School of Medicine at Dartmouth, Lebanon, NH 03766, USA. ${ }^{5}$ Department of Biological Sciences, Dartmouth College, Hanover, NH 03755, USA.

Received: 8 June 2019 Accepted: 12 November 2019

Published online: 12 December 2019

\section{References}

1. Maris JM, Hogarty MD, Bagatell R, Cohn SL. Neuroblastoma. Lancet. 2007; 369(9579):2106-20.

2. Brodeur GM. Neuroblastoma: biological insights into a clinical enigma. Nat Rev Cancer. 2003;3(3):203-16.

3. Stiller CA, Parkin DM. International variations in the incidence of neuroblastoma. Int J Cancer. 1992;52(4):538-43.

4. London WB, Castleberry RP, Matthay KK, et al. Evidence for an age cutoff greater than 365 days for neuroblastoma risk group stratification in the children's oncology group. J Clin Oncol. 2005:23(27):6459-65.

5. Brodeur GM, Pritchard J, Berthold F, et al. Revisions of the international criteria for neuroblastoma diagnosis, staging, and response to treatment. Clin Oncol. 1993;11(8):1466-77.

6. Cohn SL, Pearson ADJ, London WB, et al. The international neuroblastoma risk group (INRG) classification system: an INRG task force report. J Clin Oncol. 2009:27(2):289-97.

7. Maris JM. Recent advances in neuroblastoma. N Engl J Med. 2010;362(23): 2202-11.

8. Oppedal BR, Storm-Mathisen I, Lie SO, Brandtzaeg P. Prognostic factors in neuroblastoma. Clinical, histopathologic, and immunohistochemical features and DNA ploidy in relation to prognosis. Cancer. 1988;62(4):772-80.

9. Layfield LJ, Keith Thompson J, Dodge RK, Kerns B-J. Prognostic indicators for neuroblastoma: stage, grade, DNA ploidy, MIB-1-proliferation index, p53, HER-2/neu and EGFr-a survival study. J Surg Oncol. 1995;59(1):21-7.

10. Silber JH, Evans AE, Fridman M. Models to predict outcome from childhood neuroblastoma: the role of serum ferritin and tumor histology. Cancer Res. 1991;51(5):1426-33.

11. Bourhis J, De Vathaire F, Wilson GD, et al. Combined analysis of DNA ploidy index and N-myc genomic content in neuroblastoma. Cancer Res. 1991; 51(1):33-6.

12. Zanon C, Tonini GP. Transcription instability in high-risk neuroblastoma is associated with a global perturbation of chromatin domains. Mol Oncol. 2017;11(11):1646-58.

13. Schwab M. Amplification of N-myc as a prognostic marker for patients with neuroblastoma. Semin Cancer Biol. 1993;4(1):13-8. 
14. Huang M, Weiss WA. Neuroblastoma and MYCN. Cold Spring Harb Perspect Med. 2013;3(10):a014415.

15. Valentijn LJ, Koster J, Haneveld F, et al. Functional MYCN signature predicts outcome of neuroblastoma irrespective of MYCN amplification. Proc Natl Acad Sci. 2012;109(47):19190-5.

16. Weiss WA, Aldape K, Mohapatra G, Feuerstein BG, Bishop JM. Targeted expression of MYCN causes neuroblastoma in transgenic mice. EMBO J. 1997;16(11):2985-95.

17. Matthay KK, Maris JM, Schleiermacher G, et al. Neuroblastoma. Nat Rev Dis Primers. 2016;2(1):16078.

18. Schwab M, Westermann F, Hero B, Berthold F. Neuroblastoma: biology and molecular and chromosomal pathology. Lancet Oncol. 2003;4(8):472-80.

19. Brodeur GM, Fong CT, Morita M, Griffith R, Hayes FA, Seeger RC. Molecular analysis and clinical significance of $\mathrm{N}$-myc amplification and chromosome 1p monosomy in human neuroblastomas. Prog Clin Biol Res. 1988;271:3-15.

20. Spitz R, Hero B, Westermann F, Ernestus K, Schwab M, Berthold F. Fluorescence in situ hybridization analyses of chromosome band 1p36 in neuroblastoma detect two classes of alterations. Genes Chromosomes Cancer. 2002;34(3):299-305.

21. Attiyeh EF, London WB, Mossé YP, et al. Chromosome 1p and 11q deletions and outcome in neuroblastoma. N Engl J Med. 2005;353(21):2243-53.

22. Spitz R, Hero B, Ernestus K, Berthold F. Deletions in chromosome arms $3 p$ and $11 \mathrm{q}$ are new prognostic markers in localized and 4 s neuroblastoma. Clin Cancer Res. 2003;9(1):52-8.

23. Spitz R, Hero B, Simon T, Berthold F. Loss in chromosome 11q identifies tumors with increased risk for metastatic relapses in localized and $4 \mathrm{~S}$ neuroblastoma. Clin Cancer Res. 2006;12(11):3368-73.

24. Defferrari R, Mazzocco K, Ambros IM, et al. Influence of segmental chromosome abnormalities on survival in children over the age of 12 months with unresectable localised peripheral neuroblastic tumours without MYCN amplification. Br J Cancer. 2015;112(2):290-5.

25. Schleiermacher G, Mosseri $V$, London WB, et al. Segmental chromosomal alterations have prognostic impact in neuroblastoma: a report from the INRG project. Br J Cancer. 2012;107(8):1418-22.

26. Tonini GP. Growth, progression and chromosome instability of neuroblastoma: a new scenario of tumorigenesis? BMC Cancer. 2017;17(1):20.

27. Subramanian A, Tamayo P, Mootha VK, et al. Gene set enrichment analysis: a knowledge-based approach for interpreting genome-wide expression profiles. Proc Natl Acad Sci. 2005;102(43):15545-50.

28. Carter SL, Eklund AC, Kohane IS, Harris LN, Szallasi Z. A signature of chromosomal instability inferred from gene expression profiles predicts clinical outcome in multiple human cancers. Nat Genet. 2006;38(9):1043-8.

29. Kocak H, Ackermann S, Hero B, et al. Hox-C9 activates the intrinsic pathway of apoptosis and is associated with spontaneous regression in neuroblastoma. Cell Death Dis. 2013:4(4):e586.

30. Pugh TJ, Morozova O, Attiyeh EF, et al. The genetic landscape of high-risk neuroblastoma. Nat Genet. 2013;45(3):279-84.

31. Su Z, Fang $H$, Hong $H$, et al. An investigation of biomarkers derived from legacy microarray data for their utility in the RNA-seq era. Genome Biol. 2014;15(12):523.

32. Oberthuer A, Juraeva D, Li L, et al. Comparison of performance of one-color and two-color gene-expression analyses in predicting clinical endpoints of neuroblastoma patients. Pharmacogenomics J. 2010;10(4):258-66.

33. Bown N, Lastowska M, Cotterill S, et al. 17q gain in neuroblastoma predicts adverse clinical outcome. Med Pediatr Oncol. 2001:36(1):14-9.

34. Vandesompele J, Michels E, De Preter K, et al. Identification of 2 putative critical segments of $17 \mathrm{q}$ gain in neuroblastoma through integrative genomics. Int J Cancer. 2007;122(5):1177-82.

35. Guo C, White PS, Hogarty MD, et al. Deletion of $11 \mathrm{q} 23$ is a frequent event in the evolution ofMYCN single-copy high-risk neuroblastomas. Med Pediatr Oncol. 2000;35(6):544-6.

36. Mlakar V, Jurkovic Mlakar S, Lopez G, Maris JM, Ansari M, Gumy-Pause F. 11q deletion in neuroblastoma: a review of biological and clinical implications. Mol Cancer. 2017:16(1):114

37. White PS, Thompson PM, Seifried BA, et al. Detailed molecular analysis of 1 p36 in neuroblastoma. Med Pediatr Oncol. 2001;36(1):37-41.

38. White PS, Thompson PM, Gotoh T, et al. Definition and characterization of a region of 1 p36.3 consistently deleted in neuroblastoma. Oncogene. 2005; 24(16):2684-94.

39. Bakhoum SF, Compton DA. Chromosomal instability and cancer: a complex relationship with therapeutic potential. J Clin Invest. 2012;122(4):1138-43.
40. Grimwade D, Hills RK, Moorman AV, et al. Refinement of cytogenetic classification in acute myeloid leukemia: determination of prognostic significance of rare recurring chromosomal abnormalities among 5876 younger adult patients treated in the United Kingdom Medical Research Council trials. Blood. 2010;116(3):354-65.

41. Brodeur GM, Sekhon G, Goldstein MN. Chromosomal aberrations in human neuroblastomas. Cancer. 1977:40(5):2256-63.

42. Luttikhuis MEMO, Powell JE, Rees SA, et al. Neuroblastomas with chromosome $11 \mathrm{q}$ loss and single copy MYCN comprise a biologically distinct group of tumours with adverse prognosis. Br J Cancer. 2001;85(4):531-7.

\section{Publisher's Note}

Springer Nature remains neutral with regard to jurisdictional claims in published maps and institutional affiliations.
Ready to submit your research? Choose BMC and benefit from:

- fast, convenient online submission

- thorough peer review by experienced researchers in your field

- rapid publication on acceptance

- support for research data, including large and complex data types

- gold Open Access which fosters wider collaboration and increased citations

- maximum visibility for your research: over $100 \mathrm{M}$ website views per year

At BMC, research is always in progress.

Learn more biomedcentral.com/submissions 